\title{
Identification of key genes and pathways in Parkinson's disease through integrated analysis
}

\author{
JINGRU WANG, YINING LIU and TUANZHI CHEN \\ Department of Neurology, Liaocheng People's Hospital, Liaocheng Clinical School of Taishan Medical University, \\ Liaocheng, Shandong 252004, P.R. China
}

Received March 31, 2016; Accepted April 19, 2017

DOI: $10.3892 / \mathrm{mmr} .2017 .7112$

\begin{abstract}
Parkinson's disease (PD) is a progressive, degenerative neurological disease, typically characterized by tremors and muscle rigidity. The present study aimed to identify differentially expressed genes (DEGs) between patients with PD and healthy patients, and clarify their association with additional biological processes that may regulate factors that lead to PD. An integrated analysis of publicly available Gene Expression Omnibus datasets of PD was performed. DEGs were identified between PD and normal blood samples. Gene Ontology enrichment and Kyoto Encyclopedia of Genes and Genomes pathway analyses, as well as protein-protein interaction (PPI) networks were used to predict the functions of identified DEGs. Reverse transcription-quantitative polymerase chain reaction (RT-qPCR) was performed to validate the predicted expression levels of identified DEGs in whole blood samples obtained from patients with PD and normal healthy controls. A total of 292DEGs were identified between the PD and normal blood samples. Of these, 156 genes were significantly upregulated and 136 genes were significantly downregulated in PD samples following integrated analysis of four PD expression datasets. The 10 most upregulated and downregulated genes were used to construct a PPI network, where ubiquitin C-terminal hydrolase L1 (UCHL1), 3-phosphoinositide dependent protein kinase 1 (PDPK1) and protein kinase cAMP-activated catalytic subunit $\beta$ (PRKACB) demonstrated the highest connectivity in the network. DEGs were significantly enriched in amoebiasis, vascular smooth muscle contraction, and the Wnt and calcium signaling pathways. The expression levels of significant DEGs, UCHL1, PDPK1 and PRKACB were validated using RT-qPCR analysis. The findings revealed that UCHL1 and PDPK1 were upregulated and PRKACB was downregulated in patients with
\end{abstract}

Correspondence to: Dr Tuanzhi Chen, Department of Neurology, Liaocheng People's Hospital, Liaocheng Clinical School of Taishan Medical University, 45 Huashan Road, Liaocheng, Shandong 252004, P.R. China

E-mail: tuanzhichenlc@sina.com

Key words: integrated analysis, expression data, Parkinson's disease, microarray, differentially expressed genes
PD when compared with normal healthy controls. In conclusion, the results indicate that the significant DEGs, including UCHL1, PDPK1 and PRKACB may be associated with the development of PD. In addition, these factors may be involved in various signaling pathways, including amoebiasis, vascular smooth muscle contraction and the Wnt and calcium signaling pathways.

\section{Introduction}

Parkinson's disease (PD) is a chronic, progressive, degenerative neurological disease, and is the second most common neurodegenerative disease following Alzheimer's disease (1). The clinical symptoms of PD may be motor-associated or patients may present with non-motor manifestations. Typical motor-associated manifestations include tremors, muscle rigidity, deterioration of voluntary movements and postural instability $(2,3)$. The non-motor manifestations may include depression, autonomic dysfunction, cataracts and cognitive impairment, such as mild cognitive impairment and Parkinson's dementia (4-6).

The pathological features of PD include loss of midbrain dopaminergic neurons in the substantia nigra and intracellular inclusions of Lewy bodies predominantly composed of $\alpha$-synuclein (7). Despite fairly typical clinical and pathological features, the etiology of PD remains to be elucidated. Numerous cellular mechanisms, including oxidative stress, mitochondrial dysfunction, neuroinflammatory abnormalities and cell apoptosis have been previously reported as potential causes (8).

Oxidative stress is a key pathogenic factor in $\mathrm{PD}$. A previous study reported that the aggregation of $\alpha$-synuclein forms to soluble oligomers may interact with metal ions to induce aberrant free radical production and neuronal toxicity, leading to oxidative stress and neuronal death in PD (9). In addition, mitochondrial dysfunction may be closely associated with increased reactive oxygen species formation in PD and affects various cellular pathways, leading to damage of intracellular components and cell death (10). $\alpha$-synuclein is associated with the inner mitochondrial membrane, and may interfere with mitochondrial function by interacting with complex I and reducing mitochondrial complex I activity, which leads to increased free radical production and increased mitophagy in dopaminergic neurons $(11,12)$. 
Neuroinflammation is recognized as a pathophysiological contributor of PD. High levels of proinflammatory mediators, such as tumor necrosis factor- $\alpha$, interleukin- $1 \beta$ and interferon- $\gamma$ have been detected in the midbrain of patients with PD and contribute to the acceleration of nigral dopamine neuron degeneration $(13,14)$.

Fyn, a non-receptor tyrosine kinase, maybe involved in progressive neurodegenerative processes in PD by regulating microglial neuroinflammatory responses in $\mathrm{PD}$ in vitro and in vivo (15).

The pathogenesis of PD remains to de elucidated and currently available treatments are limited. Current treatment options for PD include levodopa, dopamine receptor agonists, acetylcholinesterase inhibitors, adenosine $2 \mathrm{~A}$ receptor antagonists, catechol-O-methyl transferase inhibitors and monoamine oxidase B-inhibitors, however those options have limited efficacy for patients with PD (16). The present study performed bioinformatics analysis to integrate the mRNA expression data from blood samples obtained from patients with PD and normal healthy controls in the Gene Expression Omnibus (GEO) database, in order to identify differentially expressed genes (DEGs). The aim of the present study was to provide valuable information for the identification of novel therapeutic targets for PD, in order to facilitate the development of effective targeted treatments.

\section{Materials and methods}

Gene expression datasets. The raw gene expression profiling datasets submitted by four individual studies (GEO accession nos. GSE54536, GSE34287, GSE18838 and GSE6613; Table I) (17-20) were obtained from the GEO database (http://www.ncbi.nlm.nih.gov/geo) (21). In order to identify differentially expressed genes in PD, four GEO datasets were downloaded and used for integrated analysis. Microarray studies investigating PD and normal blood samples were identified. Non-human studies, review articles and studies involving the integrated analysis of expression profiles were not used for the purposes of the present study.

Differential expression analysis of genes. Complete datasets from the GEO database were downloaded and preprocessed using $\log 2$ transformation and Z-score normalization in $\mathrm{R}$ (version 3.2.3; https://www.r-project.org/). The limma package (version 3.30.13; https://www.bioconductor.org/), which is a linear model for microarray data analysis (22), was used to identify DEGs by comparing the expression profiles in blood samples obtained from patients with PD with those of normal healthy controls in each individual dataset. Genes that demonstrated a P-value of $<0.01$ were considered as DEGs and were selected for further investigation.

Functional annotation of DEGs. The underlying function of DEGs was predicted using the Gene Ontology (GO) enrichment function and Kyoto Encyclopedia of Genes and Genomes (KEGG) pathway enrichment analysis $(23,24)$. A P-value of $<0.05$ and a false discovery rate (FDR) of $<0.05$ were set as the threshold values for selecting significantly enriched functional GO terms and KEGG pathways, respectively. The GENECODIS 3 online software (http://genecodis.cnb.csic. es/analysis) was used for this analysis (25-27).
Construction of protein-protein interaction (PPI) networks. Genes encoding closely interacting proteins are involved in mediating complex cellular functions. The Biological General Repository for Interaction Datasets (BioGRID, version 3.4), which is a database of known and predicted protein interactions (28), was used to screen interacting protein pairs from the identified DEGs. A PPI network involving the 10 most upregulated and downregulated DEGs was visualized using Cytoscape 3.1.0 software (http://cytoscape.org/) (29).

Reverse transcription-quantitative polymerase chain reaction $(R T-q P C R)$. A total of $5 \mathrm{ml}$ fresh whole blood samples were obtained from patients with PD ( $n=6 ; 3$ male and 3 female; age range, 52-63 years) and normal healthy controls ( $n=4 ; 2$ male and 2 female; age range, 54-64 years). Subjects were recruited from December 2015 to February 2016, in the Department of Neurology, Liaocheng People's Hospital, (Liaocheng, China). White cells were separated from whole blood samples with centrifugation at $200 \mathrm{x}$ g for $10 \mathrm{~min}$ at room temperature, and total white cell RNA was extracted using TRIzol reagent (Invitrogen; Thermo Fisher Scientific, Inc., Waltham, MA, USA). All patients provided written informed consent. The SuperScript III Reverse Transcription kit (Invitrogen; Thermo Fisher Scientific, Inc.) was used to synthesize cDNA from $1 \mu \mathrm{g}$ RNA. RT-qPCR reactions were performed using Power SYBR Green PCR Master Mix (Applied Biosystems; Thermo Fisher Scientific, Inc.), AmpliTaq Gold DNA polymerase (Applied Biosystems; Thermo Fisher Scientific, Inc.), and the Applied Biosystems 7500 Real-Time PCR System (Applied Biosystems; Thermo Fisher Scientific, Inc.). $\beta$-actin was used as an internal control for quantification of target gene expression. The relative expression of genes was calculated using the comparative $2^{-\Delta \Delta C q}$ method (30). The following PCR primers were used: Ubiquitin C-terminal hydrolase L1 (UCHL1), forward, 5'-GAAGGCCAATGTCGGGTAGAT-3', and reverse, 5'-AAAGGCATTCGTCCATCAAGTT-3'; 3-phosphoinositide dependent protein kinase 1 (PDPK1), forward, 5'-AGAGGT CAGGCAGCAACATAGAG-3' and reverse, 5'-TAAACCCTT CCGCTTATCCACT-3'; protein kinase cAMP-activated catalytic subunit $\beta$ (PRKACB), forward, 5'-TCGAGTACCTCC ATTCACTAGACCT-3', and reverse, 5'-CTGCCTTATTGT AGCCCTTGC-3'; $\beta$-actin, forward, 5'-ACTTAGTTGCGT TACACCCTT-3', and reverse, 5'-GTCACCTTCACCGTT CCA 3'. The following thermocycling conditions were used: Polymerase activation step at $95^{\circ} \mathrm{C}$ for $10 \mathrm{~min}$, followed by 45 cycles of 2-step qPCR $\left(15 \mathrm{sec}\right.$ of $95^{\circ} \mathrm{C}$ denaturation, $60 \mathrm{sec}$ of $60^{\circ} \mathrm{C}$ combined annealing and extension).

Statistical analysis. At least three independent experiments were performed for statistical evaluation. RT-qPCR experimental data are expressed as the mean \pm standard deviation. Statistical significance was determined using a Student's t-test. $\mathrm{P}<0.05$ was considered to indicate a statistically significant difference.

\section{Results}

DEGs in PD. A total of 4 mRNA expression profiles were collected (GEO accession nos. GSE54536, GSE34287, GSE18838 and GSE6613), which included the blood samples 
Table I. Details of the RNA expression profiles employed in the present study.

\begin{tabular}{lclcr}
\hline Author, year & GEO ID & \multicolumn{1}{c}{ Platform } & Samples (N:P) & Refs. \\
\hline Alieva et al, 2014 & GSE54536 & Illumina HumanHT-12 v4.0 Expression BeadChip & $5: 5$ & 17 \\
Potashkin et al, 2011 & GSE34287 & ExonHit Human Genome Wide SpliceArray 1.0 & $20: 19$ & 18 \\
Shehadeh et al, 2010 & GSE18838 & Affymetrix Human Exon 1.0 ST Array & $12: 18$ & 19 \\
Scherzer et al, 2007 & GSE6613 & Affymetrix Human Genome U133A Array & $23: 50$ & 20
\end{tabular}

GEO, Gene Expression Omnibus database; N, normal blood samples; P, blood samples from patients with Parkinson's disease.

obtained from 92 patients with PD and 60 normal healthy controls (Table I). Following normalization of the raw microarray data, 292 significant DEGs were identified inpatients with PD when compared with healthy controls. These consisted of 156 upregulated and 136 downregulated genes. Receptor interacting serine/threonine kinase 4 was the most significantly upregulated gene and mannose-P-dolichol utilization defect 1 was the most significantly downregulated gene (Table II). The 10 most significantly upregulated and downregulated genes are presented in Table II. The pattern of gene expression alterations for the 50 most significant DEGs is displayed as a heat map in Fig. 1.

GO classification of DEGs. DEGs in PD were annotated using GO database to gain insight into their putative biological roles. The threshold of GO terms was defined as $\mathrm{P}<0.05$. Enrichment analysis of the most significant biological processes is presented in Table IIIA, and included asparaginyl-tRNA aminoacylation (GO: 0006421; $\mathrm{P}=2.32 \times 10^{-7}$ ), cytotoxic $\mathrm{T}$ cell degranulation (GO: 0043316; $\mathrm{P}=2.32 \times 10^{-7}$ ) and porphyrin-containing compound metabolic processes (GO: 0006778; $\mathrm{P}=9.88 \times 10^{-6}$ ). Enrichment analysis of the most significant molecular functions is presented in Table IIIB, and included ferrochelatase activity (GO: 0004325; $\mathrm{P}=2.32 \times 10^{-7}$ ), asparagine-tRNA ligase activity (GO: 0004816; $\mathrm{P}=1.83 \times 10^{-7}$ ) and acid phosphatase activity (GO: 0003993; $\mathrm{P}=1.35 \times 10^{-6}$ ). Enrichment analysis of the most significant cellular components is presented in Table IIIC, and included eukaryotic translation initiation factor 3 complex only (GO: 0005852; $\left.\mathrm{P}=8.36 \times 10^{-5}\right)$.

Pathway enrichment analysis. KEGG pathway enrichment analysis was performed for the identified DEGs. FDR values of $<0.05$ was used as the threshold for pathway detection. The pathways that were most highly enriched were amoebiasis $\left(\mathrm{FDR}=6.92 \times 10^{-4}\right)$, vascular smooth muscle contraction $\left(\mathrm{FDR}=7.24 \times 10^{-4}\right)$ and the Wnt signaling pathway $\left(\mathrm{FDR}=8.17 \times 10^{-4}\right.$; Table IV).

PPI network construction. PPI networks of the 10 most upregulated and downregulated DEGs were constructed using Cytoscape software. The network consisted of 216 nodes and 231 edges. In the PPI network, the nodes with a high degree were classified as hub proteins, and degrees were defined to quantify the number of neighbors that a node demonstrated a direct connection with. As shown in Fig. 2, the red nodes represent upregulated genes and the green nodes represent
Table II. Significantly dysregulated genes in Parkinson's disease.

\begin{tabular}{lll} 
A, Top 10 upregulated genes & \\
\hline NCBI gene ID & Gene symbol & P-value \\
\hline 54101 & RIPK4 & $6.22 \times 10^{-7}$ \\
6369 & CCL24 & $2.42 \times 10^{-5}$ \\
5170 & PDPK1 & $3.15 \times 10^{-5}$ \\
54328 & GPR173 & $5.77 \times 10^{-5}$ \\
8111 & GPR68 & $1.46 \times 10^{-4}$ \\
57214 & KIAA1199 & $1.92 \times 10^{-4}$ \\
3699 & ITIH3 & $2.05 \times 10^{-4}$ \\
55267 & C22orf26 & $2.07 \times 10^{-4}$ \\
7345 & UCHL1 & $2.47 \times 10^{-4}$ \\
58529 & MYOZ1 & $2.71 \times 10^{-4}$
\end{tabular}

B, Top 10 downregulated genes

\begin{tabular}{llc}
\hline NCBI gene ID & Gene symbol & P-value \\
\hline 9526 & MPDU1 & $4.62 \times 10^{-6}$ \\
348995 & NUP43 & $4.91 \times 10^{-5}$ \\
5567 & PRKACB & $5.90 \times 10^{-5}$ \\
79830 & ZMYM1 & $8.01 \times 10^{-5}$ \\
10195 & ALG3 & $1.05 \times 10^{-4}$ \\
10396 & ATP8A1 & $1.30 \times 10^{-4}$ \\
2235 & FECH & $2.28 \times 10^{-4}$ \\
80169 & C17orf68 & $2.59 \times 10^{-4}$ \\
200081 & TXLNA & $2.70 \times 10^{-4}$ \\
10178 & ODZ1 & $2.84 \times 10^{-4}$ \\
\hline
\end{tabular}

NCBI, national centre for biotechnology information.

downregulated genes. The most significant hub proteins were UCHL1, PDPK1 and PRKACB (Fig. 2).

$R T-q P C R$ validation of differential expression $m R N A s$ and target genes. In order to validate the microarray analysis data, the expression levels of significant DEGs (UCHL1, PDPK1, PRKACB) were quantified by RT-qPCR analysis in 6PD and 4 normal blood control samples. No significant difference between the expression levels of UCHL1 and PDPK1 
Table III. GO function enrichment analysis of differentially expressed genes in Parkinson's disease.

A, Biological process

\begin{tabular}{|c|c|c|c|}
\hline GO ID & GO term & Count & P-value \\
\hline 0006778 & Porphyrin-containing compound metabolic process & 12 & $9.88 \times 10^{-6}$ \\
\hline 0006421 & Asparaginyl-tRNAaminoacylation & 8 & $2.32 \times 10^{-7}$ \\
\hline 0001913 & $\mathrm{~T}$ cell mediated cytotoxicity & 6 & $5.58 \times 10^{-5}$ \\
\hline 0043316 & Cytotoxic T cell degranulation & 6 & $2.32 \times 10^{-7}$ \\
\hline 0002323 & Natural killer cell activation involved in immune response & 6 & $6.98 \times 10^{-6}$ \\
\hline 043320 & Natural killer cell degranulation & 6 & $6.98 \times 10^{-6}$ \\
\hline 0006611 & Protein export from nucleus & 11 & $1.45 \times 10^{-6}$ \\
\hline 0001732 & Formation of translation initiation complex & 6 & $1.45 \times 10^{-5}$ \\
\hline 0051084 & 'De novo' post-translational protein folding & 11 & $6.37 \times 10^{-5}$ \\
\hline 0051085 & Chaperone mediated protein folding requiring cofactor & 11 & $4.49 \times 10^{-8}$ \\
\hline 0061077 & Chaperone-mediated protein folding & 11 & $1.04 \times 10^{-6}$ \\
\hline
\end{tabular}

B, Molecular function

\begin{tabular}{|c|c|c|c|}
\hline GO ID & GO term & Count & P-value \\
\hline 0004325 & Ferrochelatase activity & 6 & $2.32 \times 10^{-7}$ \\
\hline 0004816 & Asparagine-tRNA ligase activity & 8 & $1.83 \times 10^{-7}$ \\
\hline 0003993 & Acid phosphatase activity & 10 & $1.35 \times 10^{-6}$ \\
\hline 0031489 & Myosin V binding & 6 & $2.98 \times 10^{-5}$ \\
\hline 0003989 & Acetyl-CoA carboxylase activity & 8 & $3.80 \times 10^{-7}$ \\
\hline 0016421 & CoA carboxylase activity & 8 & $2.44 \times 10^{-6}$ \\
\hline 0016885 & Ligase activity, forming carbon-carbon bonds & 8 & $9.88 \times 10^{-6}$ \\
\hline 0008113 & Peptide-methionine (S)-S-oxide reductase activity & 5 & $5.45 \times 10^{-6}$ \\
\hline 0004809 & tRNA (guanine-N2-)-methyltransferase activity & 7 & $1.00 \times 10^{-7}$ \\
\hline 0016423 & tRNA (guanine) methyltransferase activity & 7 & $3.05 \times 10^{-6}$ \\
\hline 0005540 & Hyaluronic acid binding & 12 & $4.03 \times 10^{-7}$ \\
\hline
\end{tabular}

C, Cellular component

\begin{tabular}{llrr}
\hline GO ID & GO term & Count & P-value \\
\hline 0005852 & Eukaryotic translation initiation factor 3 complex & 11 & $8.36 \times 10^{-5}$ \\
\hline
\end{tabular}

GO, gene ontology.

in the PD and normal blood samples was observed (Fig. 3A and $\mathrm{B}$ ); however, the expression levels of these genes were higher in the PD samples when compared with the controls. Similarly, no significant difference in the expression levels of PRKACB between PD and normal control samples was observed; however, PRKACB expression was lower in the PD group when compared with the controls (Fig. 3C).

\section{Discussion}

The results of the present study demonstrated that UCHL1 was upregulated in PD samples, and was one of the hub proteins in the PPI network. UCHL1 encodes the 223-amino acid ubiquitin carboxyl-terminal hydrolase-L1 enzyme, which is abundant in brain tissues. Variants of the $U C H L 1$ gene are a risk factor for PD $(31,32)$. UCHL1 is primarily expressed in neurons and has been implicated in various neurodegenerative diseases, such as PD (33). Additionally, the UCHL1 protein has been identified in Lewy bodies (34). A previous study revealed that UCHL1 may be essential for the pathogenesis of PD (35). UCHL1 was associated with $\alpha$-synuclein accumulation in synaptic vesicles (36). The quantity of membrane-associated UCHL1 may affect the intracellular $\alpha$-synuclein levels and neurotoxicity. Chemical inhibition of UCHL1 by farnesylation may reduce $\alpha$-synuclein levels and improve neuronal cell viability in cellular models of $\alpha$-synuclein-associated toxicity for PD (37). Notably, the UCHL1 S18Y polymorphism has been previously associated with increased susceptibility for PD $(31,38)$. 
Table IV. Top 15 KEGG pathways obtained from enrichment analysis of differentially expressed genes in Parkinson's disease.

\begin{tabular}{|c|c|c|c|c|}
\hline KEGG ID & KEGG term & Count & FDR & Genes \\
\hline hsa05146 & Amoebiasis & 7 & $6.92 \times 10^{-4}$ & $\begin{array}{l}\text { COL4A4, IL1R2, PLCB2, COL4A1, ARG1, } \\
\text { NFKB1, PRKACB }\end{array}$ \\
\hline hsa04270 & Vascular smooth muscle contraction & 4 & $7.24 \times 10^{-4}$ & CACNA1D, PLCB2, ADCY7, PRKACB \\
\hline hsa04310 & Wnt signaling pathway & 8 & $8.17 \times 10^{-4}$ & $\begin{array}{l}\text { PPP2R5B, AXIN1, WNT16, PLCB2, MYC, } \\
\text { TBL1XR1, PPP3CB, PRKACB }\end{array}$ \\
\hline hsa04020 & Calcium signaling pathway & 5 & $8.37 \times 10^{-4}$ & $\begin{array}{l}\text { CACNA1D, PLCB2, ADCY7, PPP3CB, } \\
\text { PRKACB }\end{array}$ \\
\hline hsa04010 & MAPK signaling pathway & 10 & $8.44 \times 10^{-4}$ & $\begin{array}{l}\text { NFKB2, IL1R2, CACNA1D, NFKB1, MYC, } \\
\text { MAPKAPK3, PPP3CB, PRKACB, HSPA8, } \\
\text { FLNB }\end{array}$ \\
\hline hsa04724 & Glutamatergic synapse & 8 & $8.67 \times 10^{-4}$ & $\begin{array}{l}\text { GRIA3, GRM8, CACNA1D, PLCB2, ADCY7, } \\
\text { ADRBK2, PPP3CB, PRKACB }\end{array}$ \\
\hline hsa05200 & Pathways in cancer & 11 & $9.51 \times 10^{-4}$ & $\begin{array}{l}\text { COL4A4, NFKB2, AXIN1, WNT16, TPM3, } \\
\text { RUNX1, CSF1R, COL4A1, PML, NFKB1, } \\
\text { MYC }\end{array}$ \\
\hline hsa04970 & Salivary secretion & 6 & $9.62 \times 10^{-4}$ & $\begin{array}{l}\text { ATP1B1, TRPV6, PLCB2, ADCY7, PRKG2, } \\
\text { PRKACB }\end{array}$ \\
\hline hsa04640 & Hematopoietic cell lineage & 6 & $9.82 \times 10^{-4}$ & CD4, IL1R2, CSF1R, GP1BA, CD1C, CD44 \\
\hline hsa04540 & Gap junction & 4 & $1.02 \times 10^{-3}$ & PLCB2, ADCY7, PRKG2, PRKACB \\
\hline hsa04912 & GnRH signaling pathway & 3 & $1.32 \times 10^{-3}$ & CACNA1D, ADCY7, PRKACB \\
\hline hsa04062 & Chemokine signaling pathway & 8 & $1.39 \times 10^{-3}$ & $\begin{array}{l}\text { CCL24, ITK, PLCB2, ADCY7, NFKB1, } \\
\text { ADRBK2, CXCL12, PRKACB }\end{array}$ \\
\hline hsa04920 & Adipocytokine signaling pathway & 5 & $1.57 \times 10^{-3}$ & G6PC2, AGRP, ACACB, NFKB1, PRKAG2 \\
\hline hsa04916 & Melanogenesis & 3 & $1.60 \times 10^{-3}$ & PLCB 2, ADCY7, PRKACB \\
\hline hsa04660 & $\mathrm{T}$ cell receptor signaling pathway & 6 & $1.70 \times 10^{-3}$ & CD4, ITK, NFKB1, GRAP2, PPP3CB, CD28 \\
\hline
\end{tabular}

KEGG, Kyoto Encyclopedia of Genes and Genomes; FDR, false discovery rate.

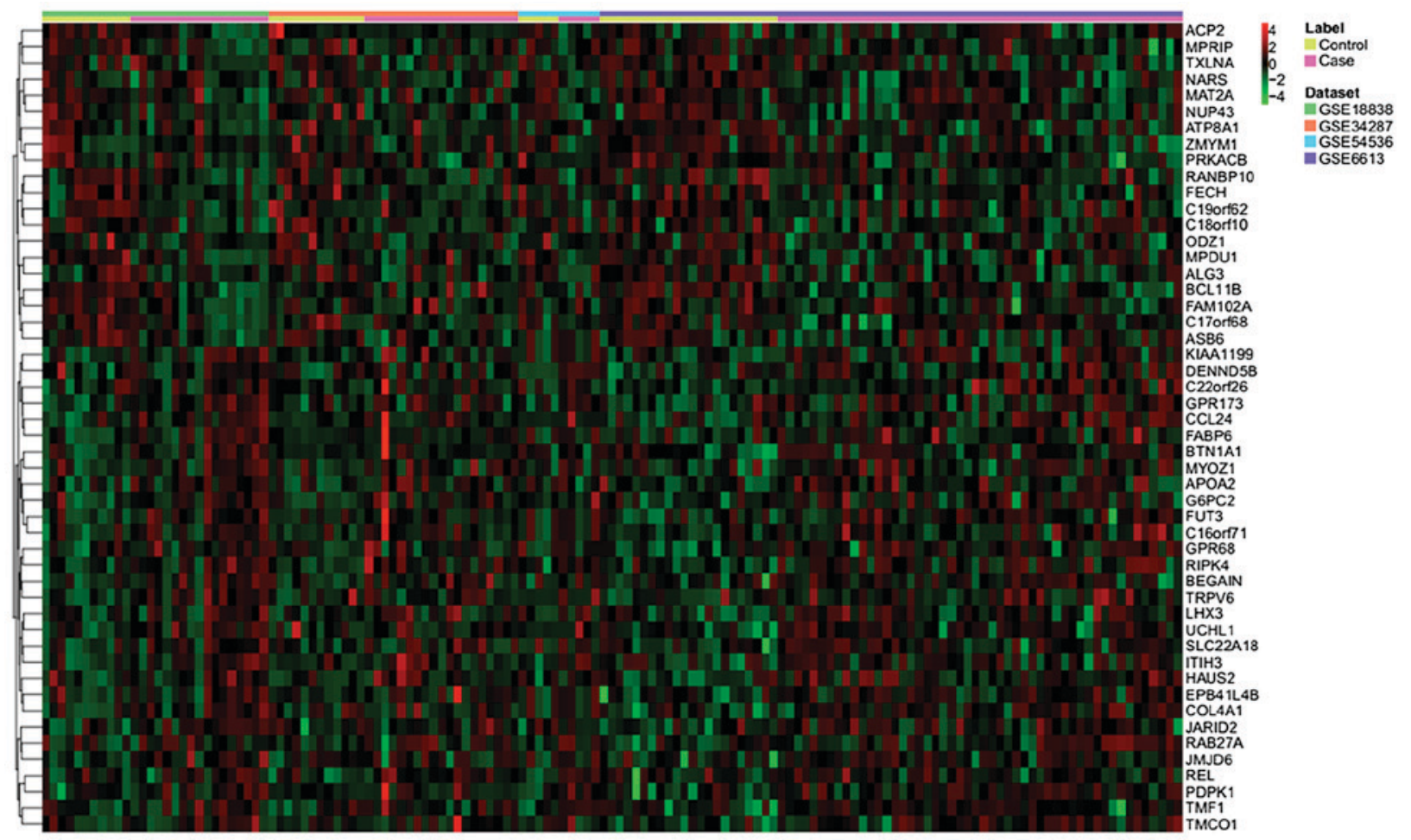

Figure 1. A heat map depicting alterations in the expression patterns of the 50 most differentially expressed genes in patients with Parkinson's disease across four different datasets (Gene Expression Omnibus accession nos. GSE18838, GSE34287, GSE54536 and GSE6613). 


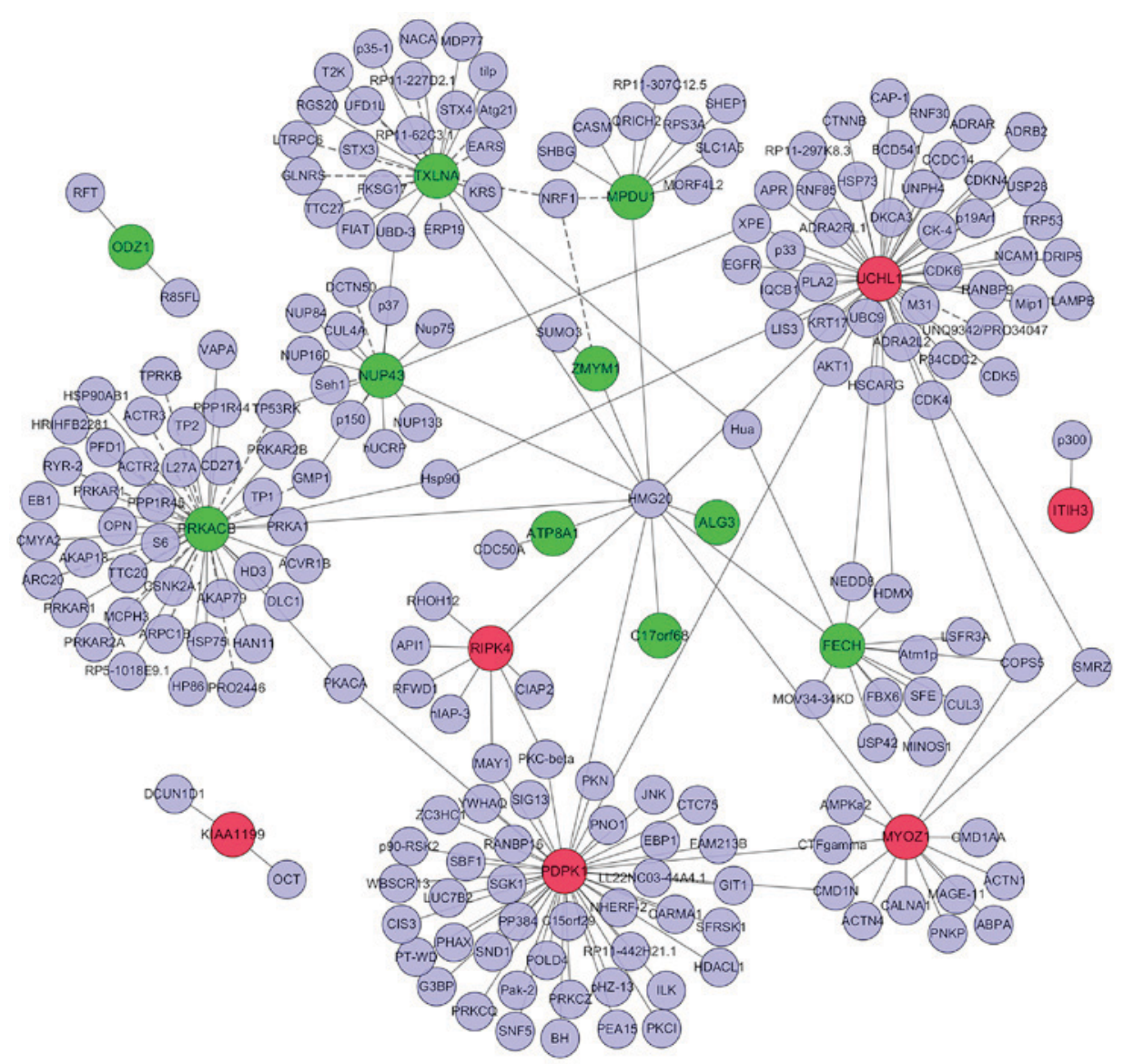

Figure 2. Protein-protein interaction networks of the 10 most significantly upregulated and downregulated DEGs. Nodes represent proteins and edges represent interactions between two proteins. Red nodes represent upregulated DEGs, whereas and green nodes indicate downregulated DEGs. Light-blue nodes represent protein products of genes predicted to interact with the DEGs. The solid lines indicate protein-protein interaction correlations, and dashed lines represent protein-protein co-localization. DEGs, differentially expressed genes.

A
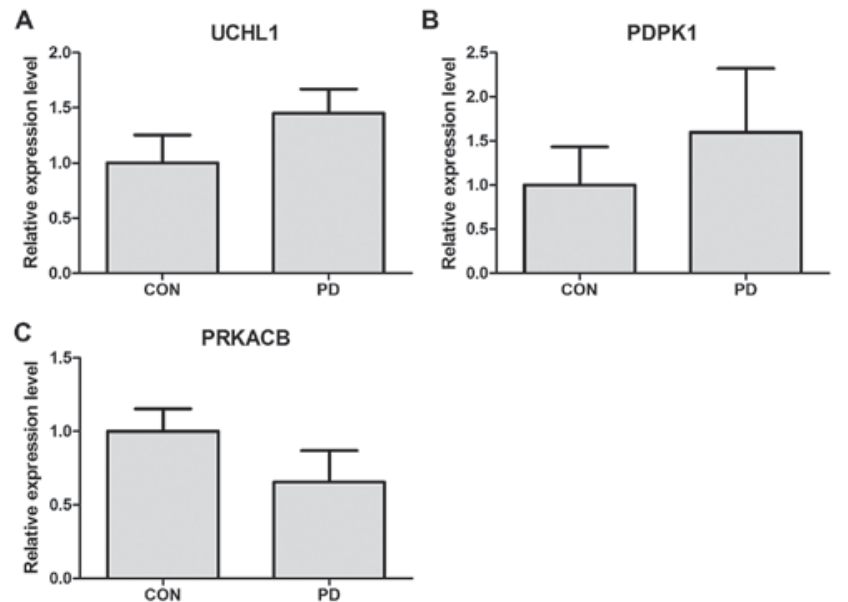

Figure 3. mRNA expression levels of (A) UCHL1 (B) PDPK1 and (C) PRKACB in PD and CON blood samples, as determined using reverse transcription-quantitative polymerase chain reaction analysis. UCHL1, ubiquitin C-terminal hydrolase L1; PDPK1, 3-phosphoinositide dependent protein kinase 1; PRKACB, protein kinase cAMP-activated catalytic subunit b; $\mathrm{CON}$, normal blood samples; $\mathrm{PD}$, blood samples from patients with Parkinson's disease.

The PDPK1 gene encodes the PDPK1 protein. Previous studies have reported that PDPK1 serves an important role in various neurodegenerative diseases, including Alzheimer's and Huntington's disease $(39,40)$. The phosphatidylinositol-4, 5-bisphosphate 3-kinase/phosphoinositide-dependent kinase (PDK)/nuclear protein kinase C/Ras-related C3 botulinum toxin pathway axis is required by the 1-42 $\beta$-amyloid peptide to induce neuronal death, and may be involved in the development of Alzheimer's disease $(41,42)$. Huntington's disease is caused by the dominant heritable expansion of atrinucleotide CAG repeat in the huntingtin gene (43). Increased levels of active PDK1 and reduced levels of extracellular-signal regulated kinase1/2 provides neuroprotection in striatal cells during Huntington's disease (44). However, the function of PDK1 in PD remains to be elucidated. The present study determined that PDK1 was upregulated in PD, and that PDK1 may therefore be involved in the development of PD.

PRKACB is a member of the serine/threonine protein kinase family and mediates signaling via cyclic adenosine monophosphate. The two catalytic subunit alleles of protein kinase A (PKA), PKAC $\alpha$ and PKAC $\beta$, are encoded by the PRKACA and PRKACB genes, respectively (45). L-dopa is an effective treatment for patients with PD; however, long-term therapy is associated with complications, such as L-dopa-induced dyskinesia. It has been previously reported that levodopa/Benserazide may prevent L-dopa-induced dyskinesia by inactivating the DR1/PKA/P- $\tau$ pathway in a rat model of PD (46). In the present study, PRAKCB was observed to be upregulated in PD samples when compared with normal 
controls, which was in accordance with the integrated analysis of mRNA expression profiles. However, the biological role of PRAKCB in PD remains to be elucidated.

In the present study, the Wnt signaling pathway was identified to be one of the significant enrichment pathways of DEGs in PD. A previous study reported that the Wnt signaling pathway potentially regulates the expression levels of the key PD protein, $\alpha$-synuclein (47). Dysregulated Wnt signaling serves an important role in the progression of PD through brain inflammation and impaired adult neurogenesis. Depletion of canonical Wnt signaling components has demonstrated a neuroprotective effect on midbrain dopaminergic neurons in a mouse model of PD (48).

In conclusion, the present study identified a total of 292DEGs in PD. The 10 most upregulated and downregulated genes were used for PPI network construction. In this network the UCHL1, PDPK1 and PRKACB genes were identified. The results suggest that these genes may serve an important role in PD via various signaling pathways, including amoebiasis, vascular smooth muscle contraction and the Wnt signaling cascade. These results may provide an important contribution to the identification of novel therapeutic targets for PD, as well as facilitate the development of effective targeted therapies. Further investigation into their biological functions may provide an additional insight into the role of the differentially regulated genes in the pathophysiology of PD.

\section{References}

1. Shulman LM: Understanding disability in Parkinson's disease. Mov Disord 25 ( Suppl 1): S131-S135, 2010.

2. Lawson RA, Yarnall AJ, Duncan GW, Khoo TK, Breen DP, Barker RA, Collerton D, Taylor JP and Burn DJ: Severity of mild cognitive impairment in early Parkinson's disease contributes to poorer quality of life. Parkinsonism Relat Disord 20: 1071-1075, 2014.

3. Wirdefeldt K, Adami HO, Cole P, Trichopoulos D and Mandel J: Epidemiology and etiology of Parkinson's disease: A review of the evidence. Eur J Epidemiol 26 (Suppl 1): S1-S58, 2011.

4. Lai SW, Lin CL, Liao KF and Chang-Ou KC: Increased risk of Parkinson's disease in cataract patients: A population-based cohort study. Parkinsonism Relat Disord 21: 68-71, 2015.

5. Lima MM, Martins EF, Delattre AM, Proenca MB, Mori MA, Carabelli B and Ferraz AC: Motor and non-motor features of Parkinson's disease-a review of clinical and experimental studies. CNS Neurol Disord Drug Targets 11: 439-449, 2012.

6. Maass A and Reichmann H: Sleep and non-motor symptoms in Parkinson's disease. J Neural Transm (Vienna) 120: 565-569, 2013

7. Hirsch EC, Jenner P and Przedborski S: Pathogenesis of Parkinson's disease. Mov Disord 28: 24-30, 2013.

8. Tolleson CM and Fang JY: Advances in the mechanisms of Parkinson's disease. Discov Med 15: 61-66, 2013.

9. Deas E, Cremades N, Angelova PR, Ludtmann MH, Yao Z, Chen S, Horrocks MH, Banushi B, Little D, Devine MJ, et al: Alpha-synuclein oligomers interact with metal ions to induce oxidative stress and neuronal death in Parkinson's disease. Antioxid Redox Signal 24: 376-391, 2016.

10. Schapira AH: Mitochondria in the aetiology and pathogenesis of Parkinson's disease. Lancet Neurol 7: 97-109, 2008.

11. Chinta SJ, Mallajosyula JK, Rane A and Andersen JK: Mitochondrial $\alpha$-synuclein accumulation impairs complex I function in dopaminergic neurons and results in increased mitophagy in vivo. Neurosci Lett 486: 235-239, 2010.

12. Greenamyre JT, MacKenzie G, Peng TI and Stephans SE: Mitochondrial dysfunction in Parkinson's disease. Biochem Soc Symp 66: 85-97, 1999.

13. Ferrari CC, Pott Godoy MC, Tarelli R, Chertoff M, Depino AM and Pitossi FJ: Progressive neurodegeneration and motor disabilities induced by chronic expression of IL-1beta in the substantia nigra. Neurobiol Dis 24: 183-193, 2006.
14. McCoy MK, Martinez TN, Ruhn KA, Szymkowski DE, Smith CG, Botterman BR, Tansey KE and Tansey MG: Blocking soluble tumor necrosis factor signaling with dominant-negative tumor necrosis factor inhibitor attenuates loss of dopaminergic neurons in models of Parkinson's disease. J Neurosci 26: 9365-9375, 2006.

15. Panicker N, Saminathan H, Jin H, Neal M, Harischandra DS, Gordon R, Kanthasamy K, Lawana V, Sarkar S, Luo J, et al: Fyn kinase regulates microglial neuroinflammatory responses in cell culture and animal models of Parkinson's disease. J Neurosci 35: 10058-10077, 2015.

16. Oertel WH: Recent advances in treating Parkinson's disease. F1000Res 6: 260, 2017.

17. Alieva AKh, Shadrina MI, Filatova EV, Karabanov AV, Illarioshkin SN, Limborska SA and Slominsky PA: Involvement of endocytosis and alternative splicing in the formation of the pathological process in the early stages of Parkinson's disease. Biomed Res Int 2014: 718732, 2014.

18. Potashkin JA, Santiago JA, Ravina BM, Watts A and Leontovich AA: Biosignatures for Parkinson's disease and atypical Parkinsonian disorders patients. PLoS One 7: e43595, 2012.

19. Shehadeh LA, Yu K, Wang L, Guevara A, Singer C, Vance J and Papapetropoulos S: SRRM2, a potential blood biomarker revealing high alternative splicing in Parkinson's disease. PLoS One 5: e9104, 2010.

20. Scherzer CR, Eklund AC, Morse LJ, Liao Z, Locascio JJ, Fefer D, Schwarzschild MA, Schlossmacher MG, Hauser MA, Vance JM, et al: Molecular markers of early Parkinson's disease based on gene expression in blood. Proc Natl Acad Sci USA 104: 955-960, 2007.

21. Edgar R, Domrachev M and Lash AE: Gene expression omnibus: NCBI gene expression and hybridization array data repository. Nucleic Acids Res 30: 207-210, 2002.

22. Diboun I, Wernisch L, Orengo CA and Koltzenburg M: Microarray analysis after RNA amplification can detect pronounced differences in gene expression using limma. BMC Genomics 7: 252, 2006.

23. Hulsegge I, Kommadath A and Smits MA: Globaltest and GOEAST: Two different approaches for gene ontology analysis. BMC Proc 3 (Suppl 4): S10, 2009.

24. Kanehisa M: The KEGG database. In 'In Silico' Simulation of Biological Processes: Novartis Foundation Symposium 247. G Bock and JA Goode (eds). John Wiley \& Sons, Ltd, Chichester, UK, pp 91-101

25. Carmona-Saez P, Chagoyen M, Tirado F, Carazo JM and Pascual-Montano A: GENECODIS: A web-based tool for finding significant concurrent annotations in gene lists. Genome Biol 8: R3, 2007.

26. Tabas-Madrid D, Nogales-Cadenas R and Pascual-Montano A: GeneCodis3: A non-redundant and modular enrichment analysis tool for functional genomics. Nucleic Acids Res 40 (Web Server Issue): W478-W483, 2012.

27. Nogales-Cadenas R, Carmona-Saez P, Vazquez M, Vicente C, Yang X, Tirado F, Carazo JM and Pascual-Montano A: GeneCodis: Interpreting gene lists through enrichment analysis and integration of diverse biological information. Nucleic Acids Res 37 (Web Server issue): W317-W322, 2009.

28. Chatr-aryamontri A, Breitkreutz BJ, Oughtred R, Boucher L, Heinicke S, Chen D, Stark C, Breitkreutz A, Kolas N, O'Donnell L, et al: The BioGRID interaction database: 2015 update. Nucleic Acids Res 43(Database issue): D470-D478, 2015.

29. Shannon P, Markiel A, Ozier O, Baliga NS, Wang JT, Ramage D, Amin N, Schwikowski B and Ideker T: Cytoscape: A software environment for integrated models of biomolecular interaction networks. Genome Res 13: 2498-2504, 2003.

30. Schmittgen TD and Livak KJ: Analyzing real-time PCR data by the comparative CT method. Nat Protoc 3: 1101-1108, 2008

31. Miyake Y, Tanaka K, Fukushima W, Kiyohara C, Sasaki S, Tsuboi Y, Yamada T, Oeda T, Shimada H, Kawamura N, et al: UCHL1 S18Y variant is a risk factor for Parkinson's disease in Japan. BMC Neurol 12: 62, 2012.

32. Göbel A, Macklin EA, Winkler S, Betensky RA, Klein C, Lohmann K and Simon DK: Genetic risk factors in Parkinson's disease: Single gene effects and interactions of genotypes. J Neurol 259: 2503-2505, 2012.

33. Ardley HC and Robinson PA: The role of ubiquitin-protein ligases in neurodegenerative disease. Neurodegener Dis 1: 71-87, 2004.

34. Lowe J, McDermott H, Landon M, Mayer RJ and Wilkinson KD: Ubiquitin carboxyl-terminal hydrolase (PGP 9.5) is selectively present in ubiquitinated inclusion bodies characteristic of human neurodegenerative diseases. J Pathol 161: 153-160, 1990. 
35. Sala G, Marinig D, Arosio A and Ferrarese C: Role of chaperone-mediated autophagy dysfunctions in the pathogenesis of Parkinson's disease. Front Mol Neurosci 9: 157, 2016.

36. Liu Y, Fallon L, Lashuel HA, Liu Z and Lansbury PT Jr: The UCH-L1 gene encodes two opposing enzymatic activities that affect alpha-synuclein degradation and Parkinson's disease susceptibility. Cell 111: 209-218, 2002.

37. Liu Z, Meray RK, Grammatopoulos TN, Fredenburg RA, Cookson MR, Liu Y, Logan T and Lansbury PT Jr: Membrane-associated farnesylated UCH-L1 promotes alpha-synuclein neurotoxicity and is a therapeutic target for Parkinson's disease. Proc Natl Acad Sci USA 106: 4635-4640, 2009.

38. Liu Y, Chen YY, Liu H, Yao CJ, Zhu XX, Chen DJ, Yang J, Lu YJ and Cao JY: Association between ubiquitin carboxy-terminal hydrolase-L1 S18Y variant and risk of Parkinson's disease: The impact of ethnicity and onset age. Neurol Sci 36: 179-188, 2015.

39. Sonoda Y, Tooyama I, Mukai H, Maeda K, Akiyama H and Kawamata T: S6 kinase phosphorylated at T229 is involved in tau and actin pathologies in Alzheimer's disease. Neuropathology 36 : 325-332, 2016

40. Naia L, Cunha-Oliveira T, Rodrigues J, Rosenstock TR, Oliveira A, Ribeiro M, Carmo C, Oliveira-Sousa SI, Duarte AI, Hayden MR and Rego AC: Histone deacetylase inhibitors protect against pyruvate dehydrogenase dysfunction in Huntington's disease 37: 2776-2794, 2017.

41. Pietri M, Dakowski C, Hannaoui S, Alleaume-Butaux A, Hernandez-Rapp J, Ragagnin A, Mouillet-Richard S, Haik S, Bailly Y, Peyrin JM, et al: PDK1 decreases TACE-mediated $\alpha$-secretase activity and promotes disease progression in prion and Alzheimer's diseases. Nat Med 19: 1124-1131, 2013.
42. Manterola L, Hernando-Rodríguez M, Ruiz A, Apraiz A, Arrizabalaga O, Vellón L, Alberdi E, Cavaliere F, Lacerda HM, Jimenez S, et al: 1-42 $\beta$-amyloid peptide requires PDK1/nPKC/Rac 1 pathway to induce neuronal death. Transl Psychiatry 3: e219, 2013.

43. A novel gene containing a trinucleotide repeat that is expanded and unstable on Huntington's disease chromosomes. The huntington's disease collaborative research group. Cell 72: 971-983, 1993.

44. Xifró X, Anglada-Huguet M, Rué L, Saavedra A,Pérez-Navarro E and Alberch J: Increased 90-kDa ribosomal S6 kinase (Rsk) activity is protective against mutant huntingtin toxicity. Mol Neurodegener 6: 74, 2011

45. Søberg K, Jahnsen T, Rognes T, Skålhegg BS and Laerdahl JK: Evolutionary paths of the cAMP-dependent protein kinase (PKA) catalytic subunits. PLoS One 8: e60935, 2013.

46. Xie CL, Wang WW, Zhang SF, Yuan ML, Che JY, Gan J, Song L, Yuan WE and Liu ZG: Levodopa/benserazide microsphere (LBM) prevents L-dopa induced dyskinesia by inactivation of the DR1/PKA/P-tau pathway in 6-OHDA-lesioned Parkinson's rats. Sci Rep 4: 7506, 2014.

47. Berwick DC and Harvey K: The importance of Wnt signalling for neurodegeneration in Parkinson's disease. Biochem Soc Trans 40: 1123-1128, 2012.

48. Dai TL, Zhang C, Peng F, Niu XY, Hu L, Zhang Q, Huang Y, Chen L, Zhang L, Zhu W, et al: Depletion of canonical Wnt signaling components has a neuroprotective effect on midbrain dopaminergic neurons in an MPTP-induced mouse model of Parkinson's disease. Exp Ther Med 8: 384-390, 2014. 